\title{
Dynamics of femtosecond laser-induced melting and amorphization of indium phosphide
}

\author{
J. Bonse, ${ }^{\text {a) }}$ S. M. Wiggins, and J. Solis \\ Instituto de Optica, CSIC, Serrano 121, 28006 Madrid, Spain
}

(Received 20 February 2004; accepted 20 May 2004)

\begin{abstract}
Laser-induced melting and resolidification of single-crystalline indium phosphide (InP) upon irradiation with $150 \mathrm{fs}$ laser pulses at $800 \mathrm{~nm}$ has been investigated by means of real-time-reflectivity measurements with subnanosecond time resolution. Melting of the surface is observed to occur very rapidly on a time scale shorter than our experimental resolution while the lifetime of the liquid phase is several tens of nanoseconds. As a result of the subsequent rapid solidification process, a thin layer of amorphous material with a thickness of several tens of nanometers is formed on the surface. The formation of this amorphous layer has been observed for every fluence above the melting and below the ablation threshold. The evolution of the reflectivity has been modeled for several different solidification scenarios and compared to the experimental results. This comparison shows that solidification proceeds interfacially from the solid interface towards the surface. A lower limit for the critical solid-liquid interface velocity for amorphization in this compound semiconductor has been estimated to be in the range of 1-4 m/s. (C) 2004 American Institute of Physics. [DOI: 10.1063/1.1771822]
\end{abstract}

\section{INTRODUCTION}

Amorphization phenomena upon irradiation of semiconductors with ultrashort laser pulses irradiation were first reported in a series of papers by Bloembergen and co-workers ${ }^{1}$ in which visible and ultraviolet picosecond laser pulses were used to irradiate single-crystalline silicon samples. Generally, such a transition between the crystalline and the amorphous phase following laser irradiation is related to a threshold behavior in the solidification velocity. If solidification proceeds with liquid-solid interface velocities higher than a critical value, irradiation leads to the amorphization of the processed material. ${ }^{2,3}$ Obviously, the actual solidification speed is strongly affected by the local temperature gradient and the transient cooling conditions after melting which are determined by the optical and thermal properties of the material phases involved and by the laser parameters (pulse duration, wavelength, spatial beam profile, etc.).

Since the pioneering work by Bloembergen and coworkers, the dynamics of femtosecond laser-induced phase transitions has been intensively studied in semiconductors such as silicon ${ }^{4,5}$ and the III-V compounds gallium $\operatorname{arsenide}^{5,6}(\mathrm{GaAs})$ and indium antimonide ${ }^{7}(\mathrm{InSb})$. In general, most of these femtosecond time resolved studies have been concentrated on the initial stages of the transformation, i.e., melting, whereas resolidification in the nanosecond time scale has been studied less intensively, in spite of the large number of works devoted to rapid solidification phenomena carried out in the 1980s in semiconductors under nanosecond laser pulses. ${ }^{8-10}$

Indium phosphide (InP) is a III-V compound semiconductor extensively used in optoelectronic applications. ${ }^{11}$ In this material the dynamics of pulsed-laser-induced melting

\footnotetext{
${ }^{\text {a) }}$ Author to whom correspondence should be addressed; electronic mail: jbonse@io.cfmac.csic.es
}

has been studied for irradiation pulses in the nanosecond regime $^{12,13}$ and more recently by femtosecond pump-probe measurements. ${ }^{14}$ This latter study as well as two previous ones, that used micro-Raman spectroscopy and atomic force microscopy to investigate the material structure after femtosecond irradiation, ${ }^{15,16}$ have reported the formation of a thin amorphous surface layer upon melting and rapid solidification of the material. In this work we report a study of the structural transformation dynamics of single-crystalline InP under femtosecond laser pulses using real-time optical measurements with subnanosecond time resolution, covering a time window up to $500 \mathrm{~ns}$. This study has provided a detailed insight into melting and rapid solidification phenomena in this material. Special attention has been paid to the determination of the solidification scenario which finally leads to the formation of the amorphous surface layer. The study has allowed us both to establish a correlation between the irradiation conditions and the characteristics of the amorphous layer formed on the surface as well as to estimate the critical liquid-solid interface speed for amorphization of single-crystalline InP.

\section{EXPERIMENTAL DETAILS}

The samples used were $400 \mu \mathrm{m}$ thick, polished (100) InP wafers (American Xtal Technology, Fremont, USA, $n$-doped). A chirped pulse amplification Ti:sapphire laser system (Spectra Physics, Spitfire), providing linearly polarized pulses of $\sim 130 \mathrm{fs}$ duration at a wavelength of $800 \mathrm{~nm}$, was used for irradiation. The duration of the laser pulses was measured using a single-shot autocorrelator (Spectra Physics, SSA) by fitting the pulses to a temporal Gaussian profile. At the irradiation wavelength (photon energy $\sim 1.55 \mathrm{eV}$ ), in the linear absorption regime, the fundamental absorption mecha- 


\section{Streak Camera Photodiode}

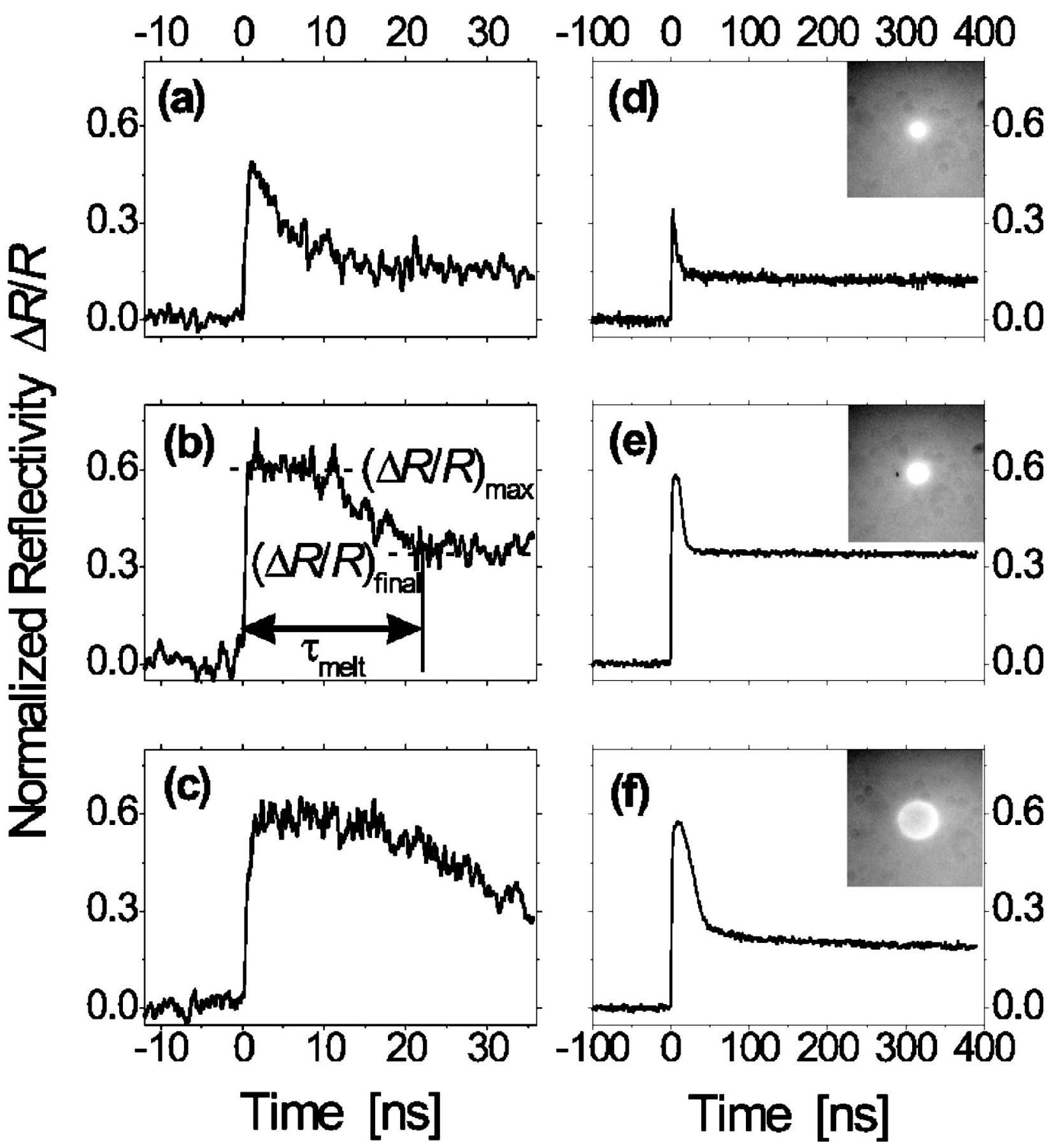

FIG. 1. Normalized surface reflectivity as a function of time as measured with streak camera (a)-(c) and with a photodiode (d)-(f) for three different pump fluence levels [(a) $\left.+(\mathrm{d}), \phi_{0} \sim 1.06 \times \phi_{\mathrm{m}} ;(\mathrm{b})+(\mathrm{e}), \phi_{0} \sim 1.14 \times \phi_{\mathrm{m}} ;(\mathrm{c})+(\mathrm{f}), \phi_{0} \sim 1.46 \times \phi_{\mathrm{m}}\right]$. The melt duration $\tau_{\text {melt }}$, maximum reflectivity change $(\Delta R / R)_{\max }$, and final reflectivity change $(\Delta R / R)_{\text {final }}$ associated with the reflectivity transients are schematically indicated in (b). The insets in (d)-(f) show optical micrographs (scale $160 \times 160 \mu \mathrm{m}^{2}$, reflectivity mode) of the laser irradiated spots, corresponding to the reflectivity curves obtained at each fluence level.

nism in crystalline InP (band gap $1.35 \mathrm{eV}$ ) is a direct interband transition. The resulting optical penetration depth $1 / \alpha$ is $\approx 300 \mathrm{~nm}$.

In the irradiation setup, the sample was placed at normal incidence in the focal plane of a lens with $150 \mathrm{~mm}$ focal length, resulting in an almost circular laser spot on the surface. The spatial profile of the irradiation beam at the sample corresponds very approximately to a Gaussian distribution with a diameter $\left(1 / e^{2}\right)$ of $\sim 120 \mu \mathrm{m}$. An $x y z$-translation stage allowed a precise positioning of the wafer after each single pulse irradiation. All irradiations were performed in air.

The temporal evolution of the surface reflectivity upon irradiation has been monitored using a single mode $s$-polarized $\mathrm{Ar}^{+}$laser operating at $514.5 \mathrm{~nm}$. This probe beam was focused at the center of the femtosecond laser irradiated region to a spot diameter $\left(1 / e^{2}\right)$ of $\sim 30 \mu \mathrm{m}$, at an angle of incidence of $18^{\circ}$. The probe beam light reflected at 


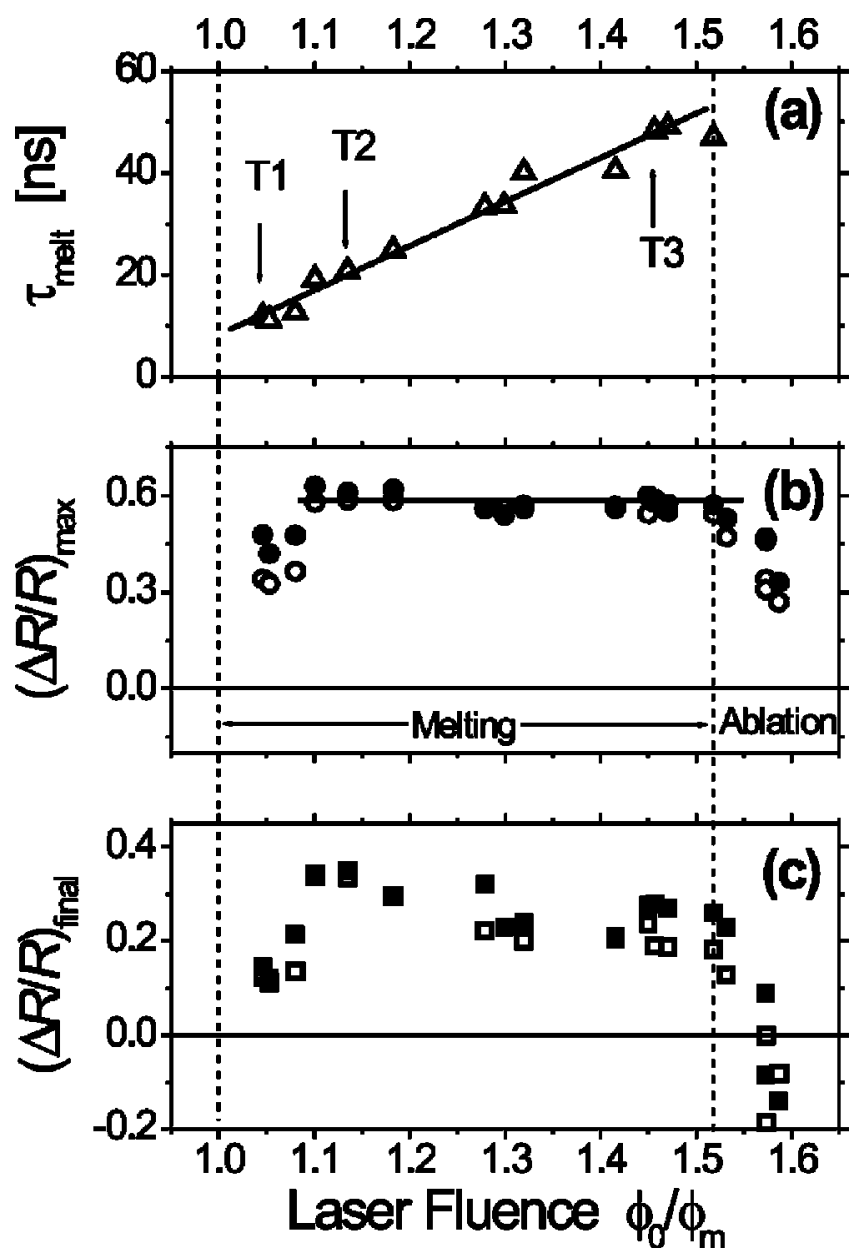

FIG. 2. Melt duration $\tau_{\text {melt }}$ (a), maximum reflectivity change $(\Delta R / R)_{\max }$ (b), and final reflectivity change $(\Delta R / R)_{\text {final }}$ (c) as function of the fluence $\phi_{0}$, normalized to the melting threshold fluence $\phi_{\mathrm{m}}$. The full symbols refer to streak camera measurements, the open symbols to photodiode measurements. The thresholds of melting and ablation are indicated by vertical dashed lines. The data points corresponding to the curves displayed in Fig. 1 are marked by symbols T1-T3.

the surface was spectrally separated from the scattered $800 \mathrm{~nm}$ radiation by means of a prism pair before simultaneous recording of its intensity evolution by means of a streak camera (Hamamatsu Model C5680, equipped with a single sweep unit Model M5676; time resolution of 350 ps in a time window of $50 \mathrm{~ns}$ ) and by a photodiode/oscilloscope detection system (a few nanoseconds temporal resolution in a time window of $500 \mathrm{~ns}$ ). Further details regarding this realtime reflectivity (RTR) setup can be found elsewhere. ${ }^{17}$

\section{RESULTS AND DISCUSSION}

Figure 1 shows the temporal evolution of the normalized surface reflectivity of the sample as measured on complementary timescales with the streak camera $[(a)-(c)]$ and with the photodiode-based detection system [(d)-(f)] upon irradiation at three different pump peak fluences $\phi_{0}$. In these curves, the reflectivity changes have been normalized to the reflectivity value of the single-crystalline material $\left[R_{C}(514.5 \mathrm{~nm})=0.36\right]$ such that $\Delta R / R$ denotes the value of $\left\{R(t)-R_{C}\right\} / R_{C}$, where $R(t)$ corresponds to the measured reflectivity at a given time $t$. Each of the selected fluences is greater than the threshold fluence for melting $\phi_{\mathrm{m}}$ but below

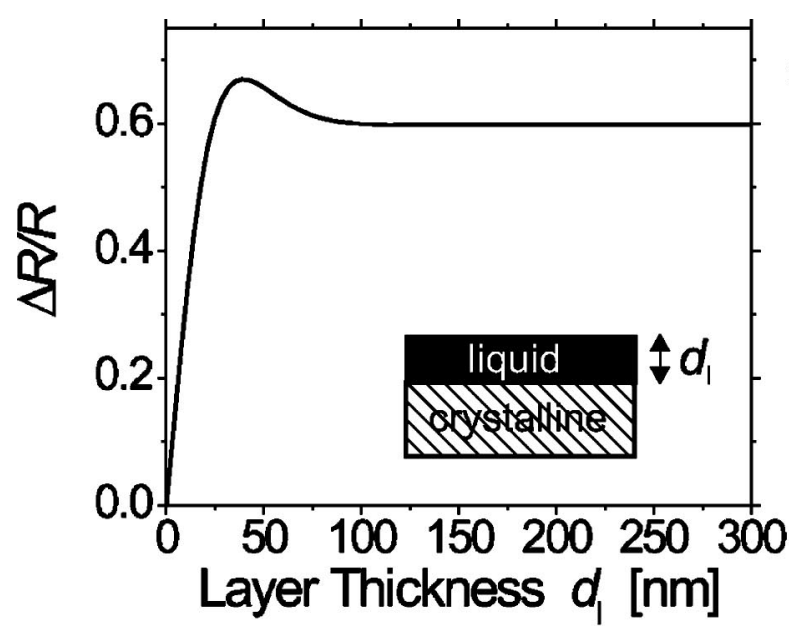

(a)

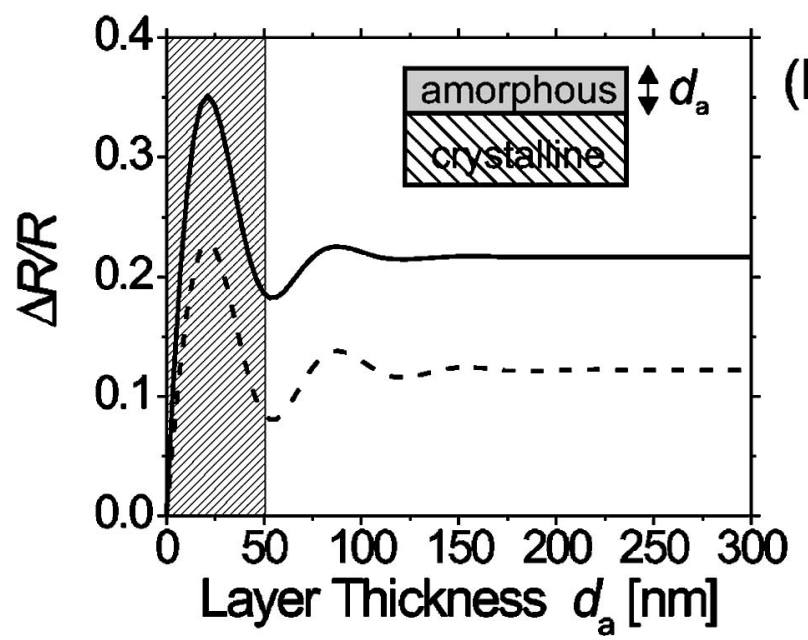

FIG. 3. Normalized surface reflectivity obtained by thin film optical simulations for (a) a molten film (liquid layer thickness $d_{l}$ ) on a crystalline substrate $(c$-InP) and (b) an amorphous film ( $a$-InP, amorphous layer thickness $d_{\mathrm{a}}$ ) on a crystalline substrate $(c$-InP). Since appropriate optical constants are not available for $l$-InP, they have been approximated by those of $l$-GaAs (Ref. 6). In (b), the dashed curve has been obtained with the optical constants $[(n+i k)(a$-InP $)=(3.9+i 1.2)]$ taken from Ref. 23 , whereas the solid curve has been obtained using an increased extinction coefficient $[(n+i k)$ $\times(a$-InP $)=(3.9+i 1.7)]$ in order to obtain optimum agreement with the experimental data shown in Fig. 2(c). The experimentally accessible amorphous thickness layer range, limited by ablation, is shaded in (b). The optical constants of $c$-InP are taken from Ref. 22.

that for ablation, $\phi_{\mathrm{abl}}=1.51 \times \phi_{\mathrm{m}}$. The measured melting and ablation thresholds are consistent within the experimental error with those given in Ref. $15\left(\phi_{\mathrm{m}}=0.16 \mathrm{~J} / \mathrm{cm}^{2}\right.$ and $\phi_{\mathrm{abl}}$ $=0.23 \mathrm{~J} / \mathrm{cm}^{2}$, respectively). The figure also shows as insets optical micrographs in reflection mode of the regions irradiated at different fluences.

At a fluence $\phi_{0} \sim 1.06 \times \phi_{\mathrm{m}}$, slightly above the melting threshold [Fig. 1(a)], the reflectivity rapidly increases to a maximum value of $(\Delta R / R)_{\max } \sim 0.48$, which is indicative of the formation of a thin molten layer at the surface. Even in the streak camera measurements, the initial rapid reflectivity increase cannot be completely temporally resolved. It has been shown by femtosecond pump-probe measurement that thermal melting of $c$-InP under very similar excitation conditions occurs within 1-2 ps with a melt front that can propagate into the material at a speed close to the speed of sound. ${ }^{14}$ After the maximum, the reflectivity decreases again to a final level of $(\Delta R / R)_{\text {final }} \sim 0.12$ after $\sim 15$ ns [Figs. 1(a) 
and $1(\mathrm{~d})$ ]. This value is consistent with the appearance of a bright spot in the optical micrograph of the laser irradiated region as shown in the inset of Fig. 1(d). The observed optical contrast is the result of the formation of a thin amorphous layer on the surface, in agreement with previous results obtained using micro-Raman spectroscopy ${ }^{15}$ and also with similar observations for femtosecond laser pulse irradiation of silicon. ${ }^{18}$ Since the whole melting and resolidification process occurs very rapidly, the values of $(\Delta R / R)_{\max }$ measured by the nanosecond resolution photodiode are somewhat smaller than those measured with the streak camera [compare Figs. 1(a) and 1(d)].

At a somewhat higher fluence of $\phi_{0} \sim 1.14 \times \phi_{\mathrm{m}}$ [Fig. 1(b)], the reflectivity rises to a reflectivity plateau with a saturated level of $(\Delta R / R)_{\max } \sim 0.60$ which is indicative of the formation of a molten layer with a thickness greater than the optical penetration depth of the probing $514.5 \mathrm{~nm}$ radiation. This molten layer resolidifies within $\sim 22 \mathrm{~ns}$ leading to a final reflectivity change of $(\Delta R / R)_{\text {final }} \sim 0.34$ [Fig. 1(e)]. The corresponding optical micrograph (see inset) likewise shows a spot with increased brightness in the center.

Further increasing of the fluence to a value just below the ablation threshold, $\phi_{0} \sim 1.46 \times \phi_{\mathrm{m}}$ [Fig. 1(c)], leads to the formation of a reflectivity plateau reaching the same saturated level of $(\Delta R / R)_{\max } \sim 0.60$ but lasting for a longer time. The total time required for complete resolidification is now $\sim 50 \mathrm{~ns}$. However, in this case, the final reflectivity level $(\Delta R / R)_{\text {final }} \sim 0.19$ measured after $\sim 400 \mathrm{~ns}$ [Fig. 1(f)] is smaller than in the previous case. A reflectivity minimum in the center of the amorphous spot is now observed in the corresponding optical micrograph [Fig. 1(f), inset].

The reflectivity transients obtained here upon nearinfrared femtosecond pulse irradiation are significantly different from those observed in Refs. 12 and 13 upon nanosecond pulse irradiation in the visible spectral range. In both nanosecond studies, two fluence-dependent reflectivity maxima were observed, in contrast to the smooth reflectivity plateau reported here. Aver'yanova et al. ${ }^{12}$ attributed the double maxima to changes of the optical properties caused by superheating of the crystal and subsequent melting, whereas Kashkarov et al. ${ }^{13}$ ascribed them to simultaneous melting and evaporation of the material. Whatever the precise mechanism upon nanosecond irradiation is, the absence of such double maxima under femtosecond irradiation (in the subnanosecond resolution transients of Fig. 1 and also in femtosecond pump-probe measurements ${ }^{14}$ ) shows that the effects stated above can be discounted in this case. The different behavior may be related to the fact that a nanosecond pulse deposits energy in both the solid and liquid phases of the material (since thermal melting occurs in a time shorter than the pulse duration) whereas a femtosecond pulse interacts only with the solid phase. However, more detailed investigation of nanosecond irradiation at fluences just above the melting threshold would be needed to clarify this issue.

Figure 2 shows the fluence dependence of several characteristic parameters that can be obtained from the time evolution of the reflectivity. They have been indicated in Fig. 1 (b) and are the melt duration $\left[\tau_{\text {melt }}\right.$, Fig. 2(a)], the maximum reflectivity change $\left[(\Delta R / R)_{\max }\right.$ Fig. 2(b)], and the final reflec- tivity change $\left[(\Delta R / R)_{\text {final }}\right.$, Fig. 2(c)] corresponding to the transients measured at different fluences. In the case of $(\Delta R / R)_{\text {final }}$ we have included the reflectivity values measured at the end of the measurement time window of the streak camera ( $t \approx 38 \mathrm{~ns}$, full symbols) and of the photodiode ( $t \approx 400 \mathrm{~ns}$, open symbols).

The melt duration $\left(\tau_{\text {melt }}\right)$ [Fig. $2(a)$ shows a linear dependence with fluence leading to values of around $50 \mathrm{~ns}$ for fluences close to the ablation threshold. The observed values (tens of nanoseconds) are not that different from those observed for nanosecond laser pulse irradiation of $c$-InP at probing wavelengths in the visible ${ }^{13}$ and infrared ${ }^{12}$ spectral regions. The linear dependence of the melt duration on the fluence is in good agreement with the 1D-thermal heat transport models summarized in Ref. 19 where, for large area irradiation with strong surface absorption (beam diameter $\gg$ energy deposition depth), at fluences slightly above $\phi_{\mathrm{m}}$, the melt duration scales linearly with the fluence.

The evolution of $(\Delta R / R)_{\max }$ steeply increases for fluences just above the melting threshold [Fig. 2(b)]. For values exceeding the threshold by only $\sim 10 \%$, a fluence independent maximum reflectivity change of $(\Delta R / R)_{\max } \sim 0.60$ can be observed which, as mentioned above, is consistent with the formation of an optically thick molten layer at the surface. For peak fluences exceeding $\sim 1.51 \times \phi_{\mathrm{m}}$ the maximum reflectivity change shows a decrease associated with the occurrence of ablation at the surface. This was confirmed by optical microscopy observations that evidenced the formation of shallow craters at the center of the irradiated regions in this fluence range.

The final surface reflectivity change $(\Delta R / R)_{\text {final }}[$ Fig. 2(c)] shows a behavior quite similar to that of the maximum transient reflectivity. For fluences slightly above the melting threshold $(\Delta R / R)_{\text {final }}$ increases to a maximum value of $\sim 0.34$. For higher fluences, it decreases smoothly to reach a nearly constant value around $\sim 0.25$ which is observed for fluences up to the ablation threshold. If the fluence is further increased, $(\Delta R / R)_{\text {final }}$ shows a strong decrease associated to the formation of a crater at the surface.

In order to understand quantitatively the time and fluence dependence of the measured reflectivity changes, they have been modeled using a multilayer scheme. ${ }^{20,21}$ The evolution of the reflectivity upon melting has been simulated by a model consisting of a layer of molten material with varying thickness $d_{1}$ on top of a crystalline (c-InP) substrate, as shown in the scheme of Fig. 3(a). Since the complex refractive index $(n+i k)$ of liquid $\mathrm{InP}(l-\mathrm{InP})$ is not known, we have used, as a reasonable approach, the values corresponding to liquid GaAs since these materials have very similar band structures in the solid phase. The values used for the simulation are thus $(n+i k)(l-\mathrm{GaAs})=(1.5+i 2.7)$ (Ref. 6) and $(n+i k)(c-\operatorname{InP})=(3.7+i 0.5) \quad($ Ref. 22) at the probing wavelength of $514.5 \mathrm{~nm}$. The simulation [Fig. 3(a)], which takes into consideration the fully coherent superposition of the Fresnel reflections at all interfaces, shows a maximum value for $\Delta R / R$ of 0.67 , corresponding to a molten film thickness of $\sim 40 \mathrm{~nm}$. This maximum corresponds to a constructive interference effect during the propagation of the melt front into the solid material. For melt depths larger than 
approximately $100 \mathrm{~nm}$, the reflectivity change saturates when the molten layer becomes optically thick, showing a value of $\Delta R / R \sim 0.60$. This saturation value is in excellent agreement with the experimental data shown in Fig. 2(b). We conclude that at this wavelength the reflectivities of bulk $l$-GaAs and $l$-InP coincide indicating that the optical constants of both liquids should be very similar at $514.5 \mathrm{~nm}$.

The comparison of the optical simulation shown in Fig. 3(a) with the experimental results of the maximum reflectivity change $(\Delta R / R)_{\max }$ shown in Fig. 2(b) also allows an estimate to be made of the thickness of the molten surface layer even for higher fluences close to the ablation threshold. Assuming a linear absorption of the femtosecond radiation according to the Lambert-Beer law and neglecting energy dissipation during the pulse, a scaling law of the molten thickness $d_{1}\left(\phi_{0}\right)=\delta \times \ln \left(\phi_{0} / \phi_{\mathrm{m}}\right)$, with a proportionality constant $\delta$ can be obtained. A value of $\delta=1.05 \mu \mathrm{m}$ is then determined from the condition that the liquid layer becomes optically thick at a fluence $\sim 10 \%$ above the melting threshold $\left[d_{1}\left(\phi_{0}=1.1 \times \phi_{\mathrm{m}}\right)=100 \mathrm{~nm}\right]$. Therefore, a melt thickness of $\sim 425 \mathrm{~nm}$ is estimated for fluences reaching the ablation threshold. This result agrees well with the value of $430 \mathrm{~nm}$ obtained from an independent estimation based on an energy balance analysis by assuming that the laser pulse energy at that fluence level is used solely for heating the solid to the melting temperature and providing the latent heat of melting.

The final reflectivity changes induced upon irradiation have been simulated by using a model consisting of a layer of $a$-InP with varying thickness $d_{\mathrm{a}}$ on top of a crystalline (c-InP) substrate as indicated in the scheme of Fig. 3(b). We have used the optical constants of $a$-InP reported by Stuke and Zimmerer $[(n+i k)(a-\operatorname{InP})=(3.9+i 1.2)]($ Ref. 23) and those of $c$-InP indicated above. The simulation shows a damped oscillatory behavior leading to a nearly constant reflectivity level of $\Delta R / R \sim 0.12$ for an amorphous layer thickness $d_{\mathrm{a}}>150 \mathrm{~nm}$ [dashed curve in Fig. 3(b)]. This level corresponds to that of bulk amorphous indium phosphide at $514.5 \mathrm{~nm}$. The reflectivity oscillations arise from interference effects of the beams being partially reflected at the air/ $a$-InP and the $a$-InP/c-InP interfaces. The initial maximum value, $\Delta R / R \sim 0.25$, reached for an amorphous layer thickness of $\sim 25 \mathrm{~nm}$, is nevertheless smaller than the experimentally observed maximum of $(\Delta R / R)_{\text {final }}$ in Fig. 2(c) ( $\sim 0.35$ for $\left.\phi_{0} \sim 1.15 \times \phi_{\mathrm{m}}\right)$. This difference is not surprising since for amorphous semiconductors, it is also well known that optical properties strongly depend on the preparation method used. ${ }^{22}$ In order to explain this difference more quantitatively we have performed additional simulations in which the optical constants of the amorphous overlayer and the underlying crystalline material were modified. They showed that an increased absorption (extinction coefficient) in recrystallized InP cannot be responsible for the experimentally observed final surface reflectivity value. The best fit of the experimental data was obtained for an amorphous layer with a refractive index $n$ of 3.9, equal to the one reported in Ref. 23 , and an increased value for the extinction coefficient of $k=1.7$. It is reasonable to assume that due to strong differences in the heating and cooling rates, the femtosecond laserinduced amorphous material can be more defective than the

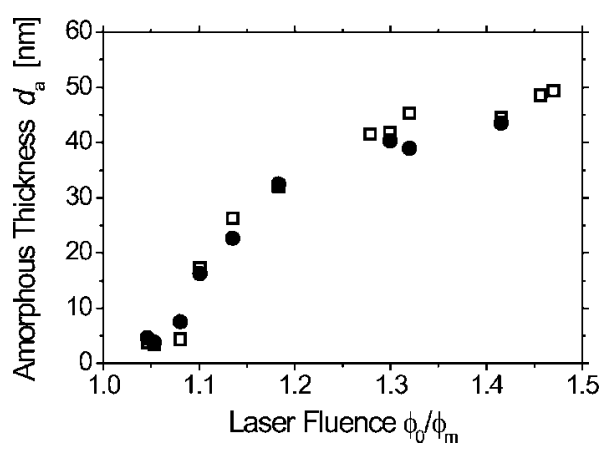

FIG. 4. Thickness of the laser-induced amorphous surface layer as a function of the fluence obtained from the streak camera (full symbols) and the photodiode (open symbols) measurements. The estimated values have been deduced by comparison of the measured final reflectivity values [Fig. 2(c)] with the simulated values [Fig. 3(b), solid curve].

evaporation based material used in the study of Stuke and Zimmerer, ${ }^{23}$ leading to the observed absorption increase.

The corresponding simulation with $(n+i k)(a$-InP $)=(3.9$ $+i 1.7)$ is shown in Fig. 3(b) with a solid line. Comparing this curve with the reflectivity values of Fig. 2(c) as a function of fluence, it is seen that both the maximum of $(\Delta R / R)_{\text {final }}$ $\sim 0.35$ and the reduced values of $\sim 0.19$ for fluences slightly below the ablation threshold are well reproduced. It is then clear that the decrease of the final reflectivity value observed in Fig. 2(c) for fluences $\phi_{0}>1.15 \times \phi_{\mathrm{m}}$ arises from the constructive interference effects being diminished when the thickness of the amorphous layer is larger than $25 \mathrm{~nm}$. Additionally, since the positions of the reflectivity maxima and minima in the simulations are not affected by the magnitude of the extinction coefficient $k$, Figs. 2(c) and 3(b) can be used to determine thickness of the amorphous layer induced upon irradiation. The corresponding result is shown in Fig. 4. For low fluences above the melting threshold, the thickness of the induced amorphous layer increases linearly with fluence. For larger fluences, the maximum thickness of amorphous material that can be produced starts to saturate (as the melt depth saturates) and is limited to $\sim 50 \mathrm{~nm}$ by the onset of ablation. This limit has been indicated in Fig. 3(b), where only the shaded region is experimentally accessible. This conclusion is additionally supported by the observation that optical micrographs of spots irradiated with a fluence slightly below the ablation threshold show only a single ring of increased reflectivity with a smooth decrease towards the center [see the inset of Fig. 1(f)]. Thicknesses of the amorphous top layer larger than $50 \mathrm{~nm}$ would imply additional reflectivity oscillations in the radial reflectivity profile which were not observed.

The formation of an amorphous top layer is similar to that observed upon nanosecond irradiation of singlecrystalline InP even if, as discussed earlier, the transient reflectivity shows different characteristics under nanosecond and femtosecond irradiation. In spatially and depth resolved photoluminescence measurements, Alferov et al. ${ }^{24}$ observed amorphous film thicknesses between 15 and $80 \mathrm{~nm}$ after irradiation of (111) InP by single 50 ns laser pulses at a wavelength of $694 \mathrm{~nm}$. This indicates that, in both cases, the 
(a)

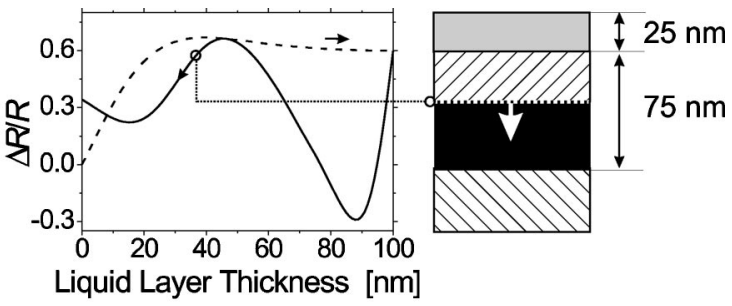

(b)

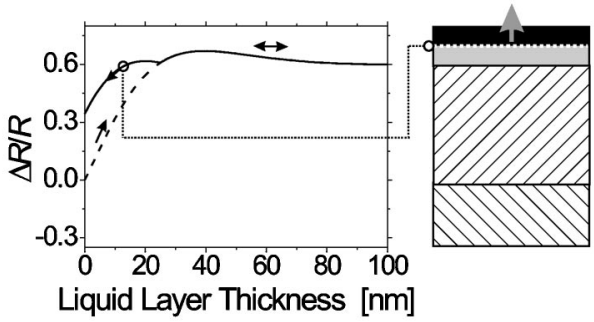

(c)

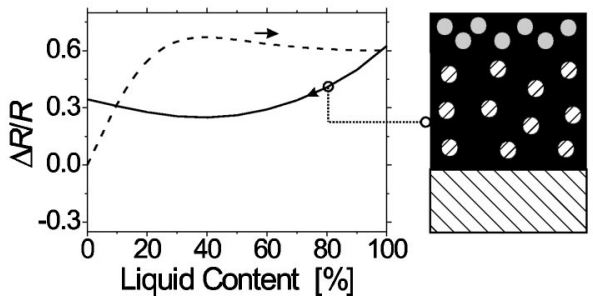

FIG. 5. Simulations of the normalized surface reflectivity for three different resolidification scenarios: after the melting of the surface (dashed curve) a $100 \mathrm{~nm}$ thick molten layer resolidifies (solid curve) into a $25 \mathrm{~nm}$ thick amorphous layer on top of a $75 \mathrm{~nm}$ thick recrystallized film via (a) interfacial resolidification from the air interface, (b) interfacial solidification from the solid interface, or (c) bulk solidification. In the corresponding schemes on the right-hand side, $l$-InP is indicated in black, $a$-InP in gray, whereas crystalline regions are shaded.

amorphization occurs from a melt pool after the laser pulse is terminated and is therefore rather insensitive to the exciting laser pulse duration.

In the previous discussion we have used optical simulations in order to analyze the reflectivity evolution upon melting and to correlate the final reflectivity changes measured with the characteristics of the amorphous layer formed at the surface. The use of optical models also allows the solidification scenario leading to the formation of the amorphous phase to be analyzed in detail. Although amorphization is generally induced upon laser irradiation through an interfacial process initiated at the solid interface, both bulk solidification ${ }^{25,26}$ and surface initiated solidification ${ }^{10,27}$ have also been reported as solidification mechanisms leading to the formation of amorphous overlayers. Therefore, in order to elucidate the solidification mechanism operating in the InP samples, we have modeled for a fluence of $\sim 1.15 \times \phi_{\mathrm{m}}$ [this is the lowest fluence that induces an optically thick molten layer as shown in Fig. 2(b)], the expected evolution of the reflectivity upon solidification of a melt layer of $100 \mathrm{~nm}$ thickness. At this fluence level, the subsequent thickness of the amorphous layer is estimated to be $25 \mathrm{~nm}$, as shown in Fig. 4, and so this layer is formed on top of a recrystallized layer of $75 \mathrm{~nm}$ thickness. The three different resolidification scenarios, indicated in Fig. 5, are (a) interfacial solidification initiated at the air interface, (b) interfacial solidification initiated at the solid interface, and (c) bulk solidification in the form of the simultaneous nucleation of spherical crystallites and amorphous regions throughout the molten volume. The dashed curve represents the interfacial melt-in curve as already discussed in Fig. 3(a), whereas the solid curves represent the results of the resolidification simulations. Their different reflectivity signatures can be used to identify the actual resolidification mechanism.

In the simulations, the interfacial solidification from the air interface leads to the appearance of a buried molten layer with decreasing thickness that solidifies into crystalline material for depths beyond $25 \mathrm{~nm}$ [Fig. 5(a), right scheme]. Interference from the light reflected at the air/a-InP, the $a$-InP/l-InP, and the $l$-InP/ $c$-InP interfaces results in a set of characteristic reflectivity oscillations in the solidification curve (solid line) with reflectivity changes showing a minimum value of $\Delta R / R \sim-0.3$. Since these large reflectivity oscillations have not been observed experimentally, this scenario can be excluded.

The interfacial solidification from the solid interface shows a different behavior [Fig. 5(b)]. Since the liquid initially resolidifies in a single-crystalline or polycrystalline state, which is here assumed to have the same optical properties as the single-crystalline material, the solidification curve (solid line) shows the same behavior as the melt-in curve (dashed line) until the $25 \mathrm{~nm}$ thick amorphous top layer is formed at the end of the process. Its formation leads to a reflectivity increase of $\sim 0.35$ with respect to the level of the nonirradiated material [in agreement with the solid curve shown in Fig. 3(b)].

In the bulk solidification scenario [Fig. 5(c)] it is assumed that spherical particles of amorphous and crystalline material are simultaneously formed in the entire liquid volume. The corresponding reflectivity evolution has been modeled by means of the Woolam V.A.S.E spectral ellipsometer software (WVASE32 Version 3.445) employing the effective medium theory of Brüggemann ${ }^{28}$ in a two-layer approach with varying liquid content. Upon solidification, the reflectivity initially drops significantly as a consequence of the simultaneous formation of the amorphous and crystalline nuclei, giving rise to the appearance of a reflectivity minimum with a value of $\Delta R / R \sim 0.25$ at a liquid content of $\sim 40 \%$. We have not observed such a minimum in the experimental evolution of reflectivity upon solidification but we cannot discard its presence due to the low noise-to-signal ratio in the measurements performed with the streak camera.

However, a clear argument for discarding the presence of a bulk solidification mechanism is given by the linear dependence of the melt duration with the laser fluence observed in Fig. 2(a). Bulk solidification is described as a process in which the solid phase nucleates at enormous rates throughout the whole volume of a strongly supercooled liquid at homogeneous temperature. ${ }^{25,29}$ Subsequently, a large amount of latent heat is rapidly released upon solidification giving rise to a strong decrease of the initial supercooling (recalescence effect) and thus to a retardation of the resolidification process. As a consequence, the evolution of the melt duration as a function of fluence is observed to show a clear discontinuity at the bulk solidification threshold. ${ }^{26,30}$ Given the absence of observable recalescence effects upon resolidification in our experimental results, we can conclude that 
solidification proceeds interfacially from the solid interface over the entire fluence range from melting to ablation.

Furthermore, since bulk solidification has been mainly observed for semiconductor films on substrates with a lower thermal conductivity than the liquid semiconductor layer leading to a heat confinement and equilibration in the melt, this interfacial solidification scenario is additionally supported by the values of the thermal conductivity of molten InP $[20-30 \mathrm{~W} /(\mathrm{m} \mathrm{K})]$ (Ref. 31) which is significantly smaller than that of the crystalline material $[70 \mathrm{~W} /(\mathrm{m} \mathrm{K})]$. $^{32}$ Consequently, the underlying crystalline material exhibits a more efficient heat flow out of the irradiated region, which prevents homogenization of the temperature in the liquid.

It is seen in Fig. 3(a) that the normalized reflectivity starts to decrease rapidly below the bulk liquid reflectivity level at a thickness of $\sim 40 \mathrm{~nm}$. Accordingly, we can use the transients in Fig. 1 to estimate the time taken by the solidification front to travel a distance equivalent to this liquid layer thickness. This time corresponds to the time elapsed between the end of the reflectivity plateau and the time at which the reflectivity reaches the amorphous solid value and ranges from 10 to approximately $30 \mathrm{~ns}$, depending on the fluence. Hence, mean interfacial front speeds between 1.3 and $4 \mathrm{~m} / \mathrm{s}$ are estimated, which represent a lower limit for the critical solid-liquid interface speed for amorphization since the interface speed is clearly not constant as most of the liquid material resolidifies in the crystalline phase and only a small part of it (the thinner upper layer) amorphizes. That is, as the liquid/solid interface propagates towards the surface, the continually higher degree of supercooling (due to heat flow and kinetic limitations ${ }^{33}$ ) in the remaining melt progressively increases the interfacial velocity until recrystallization is impeded and amorphization then occurs. The estimated critical interface velocity for InP is considerably smaller than that of single-crystalline silicon, where values between 12 and $25 \mathrm{~m} / \mathrm{s}$, depending on the crystal orientation, have been reported. ${ }^{2,34}$ This can be explained by the notion that crystalline reordering of a compound material is a more complex phenomenon than that of an elementary semiconductor. As a matter of fact, our value is in good agreement with that found for InSb, another indium containing III-V compound, for which $4-5 \mathrm{~m} / \mathrm{s}$ has been reported as the maximum crystallization interfacial speed after nanosecond laser pulse irradiation. ${ }^{3}$

\section{CONCLUSIONS}

Femtosecond laser-induced melting and solidification of single-crystalline InP has been investigated by means of realtime-resolved reflectivity measurements with subnanosecond time resolution. In the small fluence range between the melting and ablation thresholds, resolidification occurs interfacially from the solid interface giving rise to the formation of an amorphous top layer that can reach a thickness of several tens of nanometers and whose thickness is limited by the onset of ablation. The critical interface velocity for amorphization of InP has been estimated to be in the range of $1-4 \mathrm{~m} / \mathrm{s}$.

\section{ACKNOWLEDGMENTS}

This work has been partially supported by the EU in the frame of the TMR Project XPOSE (Grant No. HPRN-CT2000-00160). S.M.W. acknowledges the funding in the frame of the same project. J.B. acknowledges the funding of the CSIC through a contract in the frame of the I3P programme (Ref. I3P-PC2002), co-funded by the European Social Fund. The authors would like to thank Professor J.M. Wrobel (University of Kansas City) for his courtesy of providing the samples.

${ }^{1}$ P. L. Liu, R. Yen, N. Bloembergen, and R. T. Hodgson, Appl. Phys. Lett. 34, 864 (1979); J. M. Liu, R. Yen, E. P. Donovan, N. Bloembergen, and R. T. Hodgson, ibid. 38, 617 (1981); J. M. Liu, R. Yen, H. Kurz, and N. Bloembergen, ibid. 39, 755 (1981); R. Yen, J. M. Liu, H. Kurz, and N. Bloembergen, Appl. Phys. A: Mater. Sci. Process. 27, 153 (1982).

${ }^{2}$ A. G. Cullis, Rep. Prog. Phys. 48, 1155 (1985), and references therein.

${ }^{3}$ R. Cerny, V. Chab, G. Ivlev, E. Gatskevich, and P. Prikryl, Phys. Rev. B 59, 10685 (1999).

${ }^{4}$ C. V. Shank, R. Yen, and C. Hirlimann, Phys. Rev. Lett. 50, 454 (1983). ${ }^{5}$ K. Sokolowski-Tinten, J. Bialkowski, and D. von der Linde, Phys. Rev. B 51, 14186 (1995).

${ }^{6}$ A. M.-T. Kim, J. P. Callan, C. A. D. Roeser, and E. Mazur, Phys. Rev. B 66, 245203 (2002).

${ }^{7}$ I. L. Shumay and U. Höfer, Phys. Rev. B 53, 15878 (1996).

${ }^{8}$ D. H. Auston, J. A. Golovchenko, A. L. Simons, C. M. Surko, and T. N. C. Venkatesan, Appl. Phys. Lett. 34, 777 (1979).

${ }^{9}$ D. H. Lowndes, G. E. Jellison Jr., and R. F. Wood, Phys. Rev. B 26, 6747 (1982).

${ }^{10}$ J. J. P. Bruines, R. P. M. van Hal, H. M. J. Boots, W. Sinke, and F. W. Saris, Appl. Phys. Lett. 48, 1252 (1986).

${ }^{11}$ Properties, Processing and Applications of Indium Phosphide, edited by T. P. Pearsall (IEE Publishing, London, 2000).

${ }^{12}$ M. Y. Aver'yanova, S. Y. Karpov, Y. V. Koval'chuk, V. E. Myachin, Y. V. Pogorel'skii, V. Z. Pyataev, and I. A. Sokolov, Sov. Tech. Phys. Lett. 12, 462 (1986)

${ }^{13}$ P. K. Kashkarov, V. Y. Timoshenko, N. G. Chechenin, and A. N. Obraztsov, Laser Phys. 2, 790 (1992).

${ }^{14}$ J. Bonse, S. M. Wiggins, and J. SolisJ. Appl. Phys. (to be published).

${ }^{15}$ J. Bonse, J. M. Wrobel, K.-W. Brzezinka, N. Esser, and W. Kautek, Appl. Surf. Sci. 202, 272 (2002).

${ }^{16}$ J. Bonse, M. Munz, and H. Sturm, IEEE Trans. Nanotechnol. (in press).

${ }^{17}$ J. Solis, J. Siegel, and C. N. Afonso, Rev. Sci. Instrum. 71, 1595 (2000)

${ }^{18}$ J. Bonse, K.-W. Brzezinka, and A. J. Meixner, Appl. Surf. Sci. 221, 215 (2004).

${ }^{19}$ D. Bäuerle, Laser Processing and Chemistry, 3rd ed. (Springer, Berlin, 2000).

${ }^{20}$ F. J. Pedrotti and L. S. Pedrotti, Introduction to Optics, 2nd ed. (Prentice Hall, Englewood Cliffs, NJ, 1993).

${ }^{21}$ For details of the model see the appendix in Ref. 18.

${ }^{22}$ Handbook of Optical Constants of Solids, edited by E. D. Palik (Academic, Orlando, 1998).

${ }^{23}$ J. Stuke and G. Zimmerer, Phys. Status Solidi B 49, 513 (1972).

${ }^{24}$ Z. I. Alferov, E. N. Arutyunov, A. N. Vasil'ev, S. Y. Karpov, Y. V. Koval'chuk, V. E. Myachin, Y. V. Pogorel'skii, and I. A. Sokolov, Sov. Tech. Phys. Lett. 11, 378 (1985).

${ }^{25}$ T. Sameshima and S. Usui, J. Appl. Phys. 74, 6592 (1993).

${ }^{26}$ J. Siegel, J. Solis, C. N. Afonso, and C. Garcia, J. Appl. Phys. 80, 6677 (1996).

${ }^{27}$ J. Siegel, J. Solis, C. N. Afonso, F. Vega, J. Bankmann, O. Martínez Sacristán, and K. Sokolowski-Tinten, J. Appl. Phys. 89, 3642 (2001).

${ }^{28}$ D. A. G. Brüggemann, Ann. Phys. (Leipzig) 24, 636 (1935).

${ }^{29}$ S. R. Stiffler, M. O. Thompson, and P. S. Peercy, Phys. Rev. B 43, 9851 (1991).

${ }^{30}$ S. M. Wiggins, W. M. Gawelda, J. Solis, R. Serna, and C. N. Afonso, J. Appl. Phys. 94, 4961 (2003).

${ }^{31}$ S. Nakamura and T. Hibiya, Int. J. Thermophys. 13, 1061 (1992).

${ }^{32}$ A. Dargys and J. Kundrotas, Handbook on Physical Properties of Ge, Si, GaAs and InP (Science and Encyclopedia, Vilnius, 1994).

${ }^{33} \mathrm{M}$. von Allmen and A. Blatter, Laser-Beam Interaction with Materials, 2nd ed. (Springer, Berlin, 1995).

${ }^{34}$ P. H. Bucksbaum and J. Bokor, Phys. Rev. Lett. 53, 182 (1984). 